\title{
MEASURES OF $N$-FOLD SYMMETRY FOR CONVEX SETS ${ }^{1}$
}

\author{
CHARLES K. CHUI AND MILTON N. PARNES
}

ABSTRACT. If a convex set $S$ is 3 -fold symmetric about a point $0 \in S$, then any 3-star contained in $S$ with vertex 0 is no smaller than any other parallel 3-star contained in $S$. In this paper, among other results, we establish the converse. Consequently, we find two measures of $n$-fold symmetry, one for $n=2,3$, and the other for each $n \geqq 2$.

1. Introduction. A set $S$ in the complex plane $C$ is said to be $n$-fold symmetric about a point $P$ if every rotation about $P$ of $2 \pi / n$ maps $S$ onto itself. Let $\mathcal{F}$ be the family of all compact convex sets in $C$ with nonempty interior, that is, different from a line segment. Following Grünbaum [2], we call a real-valued function $F$ a similarity invariant measure of $n$-fold symmetry for $\mathcal{F}$, if

(i) $0 \leqq F(K) \leqq 1, K \in \mathcal{F}$;

(ii) $F(K)=1$ if and only if $K \in \mathcal{F}$ has a center of $n$-fold symmetry;

(iii) $F(K)=F(T(K))$ for every $K \in \mathcal{F}$ and every nonsingular similarity transformation $T$ of $C$ onto itself;

(iv) $F$ is continuous on $\mathcal{F}$.

There is a large literature on such measures in case $n=2$ (cf. [2]). In this paper we are going to introduce two definitions of measures for other natural numbers $n$. [See \$3.] Our first definition, which holds for $n=2,3$, comes from Theorem A. This theorem also gives a partial answer to a conjecture in the second author's dissertation [4]. Our second definition, which holds for all $n \geqq 2$, is based on Theorem B, a generalization of Hammer's result [3].

We shall use the same terminology as introduced in [5]. Let $S$ be a convex set in $C, 0 \in S$ and let $T$ be an arbitrary $n$-star at 0 contained in $S$. That is, $T$ is any $n$-star with vertex 0 and rays terminating on the boundary of $S$. Then $S$ is said to have the $n$-maximal property at 0 if every $n$-star $U$, contained in $S$ and parallel to $T$, is no larger than $T:|U| \leqq|T|$. The second author [4], [5] proved that every bounded convex set $S, n$-fold symmetric about $0 \in S$, has the $n$ -

Received by the editors October 10, 1969.

AMS 1969 subject classifications. Primary 5230.

Key words and phrases. $N$-fold symmetry, convex sets, similarity invariant measure, $n$-maximal property, $n$-supporting-line property, $n$-star.

${ }^{1}$ Some results of this paper are based on the second author's doctoral dissertation written under the direction of Professor W. Seidel. 
maximal property at 0 for every natural number $n \geqq 2$. He also conjectures that if a convex set $S$ has the p-maximal property at a point $0 \in S$, where $p$ is a prime, then $S$ should be $p$-fold symmetric about 0 . It is easy to see that the condition $p$ being a prime cannot be omitted. For if $S$ has the $n$-maximal property at 0 , it also has the $m n$-maximal property at 0 for every natural number $m$. In particular, every rectangle has the 4-maximal property at its center 0 , although it is only 2 -fold symmetric about 0 . The above conjecture is contained in the following:

ConjeCture. Let $S$ be a bounded convex set in $\mathbf{C}$ with the $n$-maximal property at $0 \in S$, and let $n=p_{1} \cdots p_{k}$, where $p_{1}, \cdots, p_{k}$ are primes. Then $S$ is $p_{j}$-fold symmetric about 0 for some $j=1, \cdots, k$.

The following theorem gives a partial answer to this conjecture.

THEOREM A. Let $G$ be a bounded convex set in $\mathcal{F}$ with the $n$-maximal property at $0 \in G$. Then if $n=2,3, G$ is $n$-fold symmetric about 0 , and if $n=4, G$ is 2-fold symmetric about 0 .

The result for $n=2$ is not new, and easily follows from a result of Hammer [3].

Let $S$ be a bounded convex set in $\boldsymbol{C}$ containing the origin 0 and let $\rho(\theta)=\rho(\theta+2 \pi),-\infty<\theta<\infty$, be the polar representation of the boundary of $S$. We say that $S$ has the $n$-supporting-line property with respect to 0 , if for every $\theta$, there are $n$ lines of support of $S$ at the angles of $\theta, \theta+2 \pi / n, \cdots, \theta+2(n-1) / n$, such that the angle between any two adjacent lines is $(n-2) \pi / n$. It is clear that if $S$ is $n$-fold symmetric about 0 , it has the $n$-supporting-line property with respect to 0 . We will prove the converse, namely

TheOREM B. Let $n \geqq 2$ be an arbitrary natural number and let $G$ be a bounded convex set in $\mathcal{F}$ having the $n$-supporting-line property with respect to an interior point 0 of $G$. Then $G$ is $n$-fold symmetric about 0 .

2. Proofs of Theorems A and B. We first prove Theorem B. Let $C$ be the boundary curve of $G$ with polar representation $\rho=\rho(\theta)$, and let $\psi=\psi(\theta)$ denote the angle at $(\theta, \rho)$ measured from the radial line to the line of support in the counterclockwise direction. Since $G$ is convex, $C$ has continuous turning tangents at all but a countable number of points, and by elementary calculus, we know that at these points

$$
\operatorname{ctn} \psi(\theta)=\rho^{\prime}(\theta) / \rho(\theta) .
$$


The $n$-supporting-line property implies

$$
\psi(\theta)=\psi(\theta+2 \pi / n)
$$

for all $\theta$. Hence, integrating $\rho^{\prime} / \rho$ and taking exponentials, we see that there exists a positive constant $c$ such that

$$
\rho(\theta)=c \rho(\theta+2 \pi / n)=\cdots=c^{n-1} \rho(\theta+2(n-1) \pi / n)=c^{n} \rho(\theta) .
$$

Hence, $c=1$ and $G$ is $n$-fold symmetric about 0 .

We shall prove Theorem $\mathrm{A}$ for $n=3$. The proofs for $n=2,4$ are similar and easier. We first list the following five geometric observations, the proofs of which are quite elementary.

(1) Let $\Delta$ be an equilateral triangle with center 0 and interior $G$. Let $T$ be a 3-star contained in $G$ with vertex 0 , and let $U$ be any 3star contained in $G$ and parallel to $T$. Then $|U| \leqq|T|$ and equality holds if and only if either the rays of $U$ fall on different sides of $\Delta$ or $U=T$.

(2) Let $T$ be a 3-star of finite length, and let $r_{1}, r_{2}, r_{3}$ be its rays. Let $U$ be another 3 -star parallel to $T$ with rays $\tilde{r}_{1}, \tilde{r}_{2}, \tilde{r}_{3}$ such that the vertex of $U$ lies on $r_{3} ; r_{1}, r_{2}, \tilde{r}_{1}, \tilde{r}_{2}$ terminate on a common straight line; and $r_{3}, \tilde{r}_{3}$ terminate at the same point. Then $|T| \leqq|U|$, and equality holds if and only if $T=U$.

(3) Let $T$ be a 3-star with rays $r_{1}, r_{2}, r_{3}$ and let $r_{1}, r_{2}$ terminate on straight lines $L_{1}, L_{2}$ respectively, where either $L_{1}$ and $L_{2}$ are parallel or they intersect on the same side of the line passing through the tips of $r_{1}, r_{2}$ as $r_{3}$. Then it is possible to construct a 3-star $U$ with rays $\tilde{r}_{1}$, $\tilde{r}_{2}, \tilde{r}_{3}$ parallel to $r_{1}, r_{2}, r_{3}$ respectively, such that $\tilde{r}_{j}$ terminates on $L_{j}$, $j=1,2 ; r_{3}$ and $\tilde{r}_{3}$ terminate at the same point; $r_{3} \subset \tilde{r}_{3} ;$ and $|T|<|U|$.

(4) Let $T$ be a 3 -star contained in a triangle $\Delta$ such that the vertex of $T$ lies in the interior of $\Delta$ and each ray of $T$ terminates at an interior point of a different side of $\Delta$. Construct the three equilateral triangles $\Delta_{1}, \Delta_{2}, \Delta_{3}$ such that one side of each $\Delta_{j}$ lies on an extended side of $\Delta$ and the other two sides of $\Delta_{j}$ pass through the other two tips of the rays of $T$. Then at least one of $\Delta_{1}, \Delta_{2}, \Delta_{3}$ contains $T$ in its interior.

(5) Let $\Delta$ be a triangle which is not equilateral, and let $T$ be a 3-star contained in $\Delta$ such that the vertex of $T$ lies in the interior of $\Delta$ and each ray of $T$ terminates at an interior point of a different side of $\Delta$. Then there is a 3-star $U$ contained in $\Delta$ and parallel to $T$ such that the vertex of $U$ is arbitrarily close to that of $T$ and $|U|>|T|$.

The proofs of (1) through (4) are quite straightforward, while the proof of (5) follows from (1) through (4). As a corollary of these observations, we obtain 
TheOREM 2.1. Let $G$ be the interior of a convex polygon $P, T$ a 3-star with vertex in $G$ and contained in $G$ such that $|T| \geqq|U|$ where $U$ is any 3 -star contained in $G$ and parallel to $T$. Then either at least one ray of $T$ terminates at a vertex of $P$ or the three rays of $T$ terminate on the interior of three different sides of $P$, which, when extended, form an equilateral triangle that contains $T$ in its interior.

Proof. Suppose that the three rays of $T$ terminate on the interior of the sides of $P$. By (2) these rays terminate on different sides, and by (3) these three sides, when extended, form a triangle which contains $G$ in its interior. By (5) this triangle is indeed equilateral.

We remark that for the special case of convex polygons Theorem A is already proved. Indeed, from the above proof we see that 3-maximal property implies 3-supporting-line property which in turn, by Theorem B, implies 3-fold symmetry. To include a larger collection of convex sets, we need the following

Lemma 2.1. Let $G$ be a convex domain with boundary $C$. Let $T$ be a 3-star with vertex $0 \in G$ and rays terminating on $C$ at points where $C$ has continuous turning tangents, such that $T$ is no smaller than any parallel 3-siar contained in $G$. Then the lines of support $L_{1}, L_{2}, L_{3}$ of $G$ at the tips $a_{1}, a_{2}, a_{3}$, respectively, of $T$ form an equilateral triangle.

Proof. The lines of support form one of the configurations as described in our above five observations. For instance, assume that they form a triangle, which is not equilateral, such that $T$ terminates on the interior of its three different sides as in observation (4). Let $L_{1}$ $=L_{1}^{\prime}$ be the line of support on which an equilateral triangle as described in the conclusion of (4) can be constructed. Let $L_{2}^{\prime}$ and $L_{3}^{\prime}$ be drawn through $a_{2}$ and $a_{3}$ respectively to form this equilateral triangle. One can find a 3 -star $T^{*}$ contained in $G$, parallel to $T$ and with vertex $0^{*} \in G$ arbitrarily close to 0 so that the rays of $T^{*}$ intersect $L_{2}^{\prime}$ and $L_{3}^{\prime}$ in $G$ or on $C$. [Cf. Fig. a or Fig. b.] Using the fact that a tangent line to a curve is a much closer approximation to the curve than any secant line, one can easily see that if $0^{*}$ is suitably chosen $\left|T^{*}\right|>|T|$. This is a contradiction. The proofs for the other configurations are similar.

We can now complete the proof of Theorem A $(n=3)$. Let $C$ be the boundary curve of $G$. Then $C$ has continuous turning tangents at all but a countable number of points. Hence, by using the right-hand derivatives and Lemma 2.1, $G$ has the 3-supporting-line property with respect to 0 , and is, therefore, 3 -fold symmetric about 0 by Theorem B. 


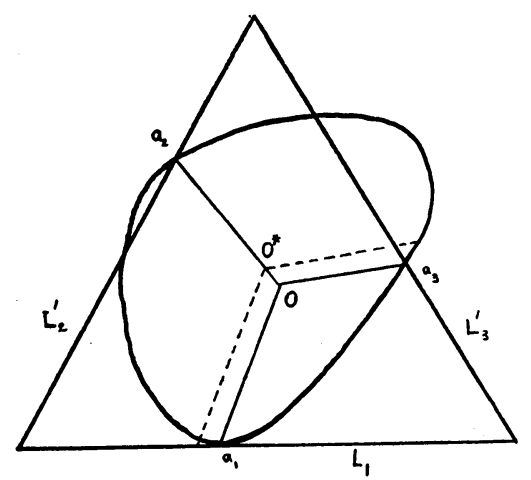

FIGURe a

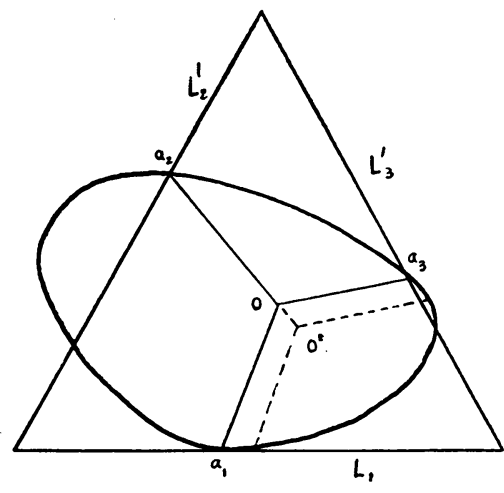

FIGURE $b$

3. Measures of symmetry. Let $G \in \mathcal{F}, P$ a point in $G$ and let $P_{\theta}$, $0 \leqq \theta<2 \pi / n$, denote the $n$-star contained in $G$, with vertex $P$, and having a ray with an angle of inclination $\theta$ measured positively from the real axis. Let

$$
M_{n}(G ; P)=\inf _{Q, \theta} \frac{\left|P_{\theta}\right|}{\left|Q_{\theta}\right|},
$$

where $Q$ runs over $G$ and $0 \leqq \theta<2 \pi / n$. We define the function $M_{n}$ on $\mathcal{F}$ by

$$
M_{n}(G)=\sup _{P \in G} M_{n}(G ; P), \quad G \in \mathcal{F} .
$$

THEOREM 3.1. For $n=2,3, M_{n}$ is a similarity invariant measure of n-fold symmetry for $\mathcal{F}$.

Proof. It is clear that $M_{n}$ satisfies (i) in $\$ 1$. Since each $G \in \mathcal{F}$ has nonempty interior, the useful $n$-stars $Q_{\theta}$ in the definition of $M_{n}(G ; P)$ have lengths bounded away from zero; hence, $M_{n}$ is continuous. Finally, for $n=2,3$, we see that, using Theorem $\mathrm{A}, M_{n}(G)=1, G \in \mathcal{F}$, if and only if $G$ is $n$-fold symmetric.

Note that in defining $M_{n}$, we have required that the sets $G$ to have nonempty interior, that is, to be different from a line segment. In the latter case, it is not clear how to interpret $\left|P_{\theta}\right| /\left|Q_{\theta}\right|$, when both $\left|P_{\theta}\right|$ and $\left|Q_{\theta}\right|$ are zero for all, except one, values of $\theta, 0 \leqq \theta<2 \pi / n$. Indeed, in this case, continuity for $M_{3}$ breaks down: If we approximate a line segment $L$ by rectangles, the limit would be $\frac{1}{2}$; however, if we approximate $L$ by isosceles triangles, it would be $2 / 5$.

We now define our second measure based on Theorem B. Let 
$G \in \mathcal{F}, P$ a point in $G$ and $P_{\theta}$ as defined above, $0 \leqq \theta<2 \pi / n$. Let $\Delta$ be a regular $n$-gon such that $G$ is contained in the closure of its interior and such that if $\Delta^{\prime}$ is another regular $n$-gon which lies in the interior of $\Delta$, then $G$ does not lie in the closure of the interior of $\Delta^{\prime}$. Such a $\Delta$ will be called admissible. Let $Q$ be the center of $\Delta$. (If $n=2, \Delta$ is a pair of parallel lines and $Q$ is equidistant from these two lines.) Let $Q_{\theta}$ be the $n$-star contained in the interior of $\Delta$ with vertex $Q$ and parallel to $P_{\theta}$. We define

$$
N_{n}(G ; P)=\inf _{\Delta} \sup _{\theta} \frac{\left|P_{\theta}\right|}{\left|Q_{\theta}\right|},
$$

where $0 \leqq \theta<2 \pi / n$ and $\Delta$ runs over all admissible regular $n$-gons. We now define the function $N_{n}$ on $\mathcal{F}$ by

$$
N_{n}(G)=\sup _{P \in G} N_{n}(G ; P), \quad G \in \mathcal{F} .
$$

THEOREM 3.2. For each natural number $n \geqq 2, N_{n}$ is a similarity invariant measure of $n$-fold symmetry for $\mathcal{F}$.

Proof. For $G \in \mathcal{F}$, it is obvious that $0 \leqq N_{n}(G) \leqq 1$. If $G$ has a positive diameter $d$, each admissible $\Delta$ has diameter no less than $d$. Hence, the corresponding $Q_{\theta}$ has length bounded away from $d$. Continuity of $N_{n}$ follows. By Theorem B, it is not difficult to see that $N_{n}(G)=1, G \in \mathcal{F}$, if and only if $G$ is $n$-fold symmetric.

Note that different from $M_{n}\left(n=3\right.$, say), the continuity of $N_{n}$ even holds for a line segment $L$. In fact, approximating $L$ by sets in $\mathcal{F}$, we should define $N_{2}(L)=1$ and $N_{3}(L)=\frac{1}{2}$. Next, we find a lower bound of $M_{3}(G), G \in \mathcal{F}$, larger than zero. We need the following lemma (cf. [1]).

LEMMA 3.1. Every convex set of diameter $d$ is contained in a circle of diameter no greater than $2 d / \sqrt{ } 3$.

THEOREM 3.3. For each $G \in \mathcal{F}, 1 / 2 \sqrt{ } 3 \leqq N_{3}(G) \leqq 1$.

Proof. For an admissible $\Delta$, let $Q_{\theta}$ be the corresponding 3-star parallel to $P_{\theta}$. Using Lemma 3.1 and elementary geometry, we see that

$$
\sup _{\theta} \frac{\left|P_{\theta}\right|}{\left|Q_{\theta}\right|} \geqq \frac{1}{2 \sqrt{ } 3}
$$

for every admissible $\Delta$. Hence, the result follows. 


\section{REFERENCES}

1. H. G. Eggleston, Convexity, Cambridge Tracts in Math. and Math. Phys., no. 47, Cambridge Univ. Press, New York, 1958, p. 111. MR 23 \#A2123.

2. B. Grünbaum, Measures of symmetry for convex sets, Proc. Sympos. Pure Math., vol. 7, Amer. Math. Soc., Providence, R. I., 1963, pp. 233-270. MR 27 \#6187.

3. P. C. Hammer, Diameters of convex bodies, Proc. Amer. Math. Soc. 5 (1954), 304-306. MR 15, 819.

4. M. Parnes, Symmetrization and conformal mapping, Ph.D. thesis, Wayne State Univ., Detroit, Mich., 1968.

5. - A distortion theorem for doubly connected regions, Proc. Amer. Math. Soc. 26 (1970), 85-91.

State University of New York at Buffalo, Amherst, New York 14226 